\title{
Hypoxic conditioned medium from mesenchymal stem cells promotes lymphangiogenesis by regulation of mitochondrial-related proteins
}

\author{
Chang Youn Lee ${ }^{1 \dagger}$, Jin Young Kang ${ }^{2 \dagger}$, Soyeon $\mathrm{Lim}^{3}$, Onju Ham ${ }^{4}$, Woochul Chang ${ }^{5^{*}}$ and Dae-Hyun Jang ${ }^{6^{*}}$
}

\begin{abstract}
Background: Recently, cell-based therapeutic lymphangiogenesis has emerged and provided hope for lymphatic regeneration. Previous studies have demonstrated that secretomes of mesenchymal stem cells (MSCs) facilitate the regeneration of various damaged tissues. This study was conducted to evaluate the lymphangiogenic potential of hypoxic conditioned media (HCM) from MSCs.

Methods: To investigate the effects of MSC-secreted factors in starved human lymphatic endothelial cells (hLEC), hLECs were treated with endothelial basal medium (EBM)-2 (control), normoxic conditioned media (NCM), or HCM in vitro and in vivo.

Results: MSCs expressed lymphangiogenic factors including EGF, FGF2, HGF, IGF-1, and VEGF-A and -C. hLECs were treated with each medium. hLEC proliferation, migration, and tube formation were improved under HCM compared with NCM. Moreover, expression of mitochondrial-related factors, MFN1and 2, were improved in HCM-treated hLECs. Lymphedema mice injected with HCM showed markedly decreased lymphedema via increased lymphatic vessel formation when compared with EBM-2- or NCM-treated mice.

Conclusions: This study suggested that HCM from MSCs contain high levels of secreted lymphangiogenic factors and promote lymphangiogenesis by regulating mitochondrial-related factors. Thus, treatment with HCM may be a therapeutic strategy for lymphedema.
\end{abstract}

Keywords: Hypoxic conditioned media, Mesenchymal stem cells, Mitochondrial-related protein, Lymphangiogenesis, Lymphatic endothelial cells

\section{Background}

Lymphedema is a pathologic swelling (edema) that results from the accumulation of protein-rich fluid in the interstitial space because of congenital or acquired lymphatic system damage [1]. Secondary lymphedema is caused by disruption or obstruction of the normal lymphatic system in response to infection, trauma, or

\footnotetext{
*Correspondence: wchang1975@pusan.ac.kr; dhjangmd@naver.com ${ }^{\dagger}$ Equal contributors

${ }^{5}$ Department of Biology Education, College of Education, Pusan National University, Busan 46241, Republic of Korea

${ }^{6}$ Department of Rehabilitation Medicine, Incheon St. Mary's Hospital, College of Medicine, The Catholic University of Korea, Dongsu-ro 56, Bupyeong-gu, Incheon 21431, Republic of Korea

Full list of author information is available at the end of the article
}

iatrogenic processes such as surgery (radical lymph node dissection) or radiation-related cancer therapy [2]. Secondary lymphedema is a lifelong condition that disturbs quality of life with signs and symptoms such as heaviness, discomfort, and impaired mobility of the limbs as well as a weakened immunity. To date, the only proposed treatment options have been pharmacotherapy, physiotherapy, and surgical treatments, such as lymph node transfer and lymphatic bypass. However, these methods require good compliance and lifelong care [3]. Recently, cell-based therapy for therapeutic lymphangiogenesis has emerged and made lymphatic regeneration possible [4]. Essential lymphatic growth factors, including epidermal growth factor (EGF), fibroblast growth 
factor (FGF), hepatocyte growth factor (HGF), insulinlike growth factor (IGF)-1, vascular endothelial growth factor (VEGF)-A, and VEGF-C, have been identified in several studies [5-10]. In addition, these growth factors have been shown to contribute to lymphangiogenesis in the damaged lymphatic areas [11].

Mesenchymal stem cells (MSCs) are multipotent cells that can be obtained from adult donors and are known to have a low risk of immune rejection [12]. Therefore, therapeutic applications using MSCs have been extensively studied and applied to various tissues in regenerative medicine owing to these beneficial effects. Moreover, MSC therapy has recently been introduced to the market. However, limitations such as poor survival, limited differentiation, and dedifferentiation of cells with passaging and donor site morbidity exist [13-16]. To avoid these limitations, researchers have turned their attention to new therapeutic approaches. MSCs are known to secrete various cytokines and growth factors, which show paracrine and autocrine activities for injured cells, particularly for those in hypoxic, apoptotic, or inflamed areas $[17,18]$. The secreted factors have been demonstrated to have many beneficial therapeutic effects in various diseases, such as neurodegenerative diseases, cancers, and heart failure [19]. Several reports have shown that cytokines secreted from MSCs under normal growth conditions can promote lymphangiogenesis via VEGF-A and VEGF-C [20-22]. MSC-based therapy has been suggested to be the most promising stem cell therapy for lymphangiogenesis [4].

Some researchers showed the possibility of regulating stem cell paracrine actions via different culture methods [18]. As one of the modifications, MSCs exposed to hypoxia showed more protein secretion and greater paracrine effects. MSCs under hypoxia also showed increased proliferation and migration compared with those under normal growth conditions, and treatment with conditioned media from hypoxic MSCs exerted therapeutic effects on wound healing by enhancing the production of angiogenic paracrine factors including basic FGF, IGF, and VEGF [23]. Another study reported that conditioned media obtained from MSCs under hypoxia showed protective effects against cardiomyoblasts in hypoxia and angiogenic effects on endothelial cells [24].

Mitochondria are important organelles that maintain cellular homeostasis under stressful conditions, such as apoptotic stimuli, an increase in reactive oxygen species (ROS), and changes in intracellular calcium concentration [25-27]. To maintain their function, mitochondria continuously undergo fission and fusion. Mitofusins MFN1 and MFN2 in the mitochondrial outer membrane have been shown to cause mitochondrial membrane fusion by binding with OPA1 in the inner membrane, whereas dynamin-related protein 1 is mainly involved with mitochondrial fission based on the phosphorylation status [28, 29]. Recent studies demonstrated that MFNs are necessary for angiogenic function in endothelial cells $[30,31]$. In addition, VEGF-A is an important factor for angiogenesis, which can stimulate the activation of MFNmediated signaling pathways [31]. We hypothesized that MFNs also play important roles in lymphangiogenesis.

We investigated the therapeutic ability of hypoxic conditioned media (HCM) from MSCs for lymphatic edema using in vitro and in vivo experimental systems. Higher expression of VEGF, a lymphangiogenic factor, was observed in hypoxic MSCs, and tube formation increased in human lymphatic endothelial cells (hLECs) treated with HCM. Overall, we confirmed that HCM have the ability to induce lymphangiogenesis in hLECs and electrocauterized mice.

\section{Methods \\ Cell culture}

Human bone marrow MSCs (hMSCs; catalogue number PT-2501) and adult human dermal lymphatic microvascular endothelial cells (hLECs) (HMVECs-dLyAd; catalogue number CC-2810) were purchased from Lonza (Basel, Switzerland). hMSCs were maintained at $37^{\circ} \mathrm{C}$ in a humidified atmosphere containing $5 \% \mathrm{CO}_{2}$. Culture media was composed of $10 \%$ fetal bovine serum (Invitrogen, Waltham, MA, USA), Dulbecco's modified Eagle's medium-low glucose, $100 \mathrm{U} / \mathrm{ml}$ penicillin (Invitrogen), and $100 \mu \mathrm{g} / \mathrm{ml}$ streptomycin (Invitrogen). Media were replaced every 3 days. We used 7-10 passages of hMSCs for experiments. hLECs were cultured in Lonza EGM-2MV medium and replaced with fresh media every 2 days.

\section{HCM from MSCs}

hMSCs were incubated for 1 day after they were seeded in a $100 \mathrm{~mm}$ dish $\left(1 \times 10^{6}\right.$ cells/dish), washed twice with endothelial basal medium (EBM-2; Lonza), and then placed into a hypoxic chamber (Anaerobic Environment; ThermoForma, Waltham, MA, USA) containing $5 \mathrm{ml}$ EBM-2 for 12 hours. The airtight humidified hypoxic chamber was maintained at $37{ }^{\circ} \mathrm{C}$ and continuously supplied with mixed gas $\left(5 \% \mathrm{CO}_{2}, 10 \% \mathrm{H}_{2}\right.$, and $\left.85 \% \mathrm{~N}_{2}\right)$. The oxygen level in the chamber was $\sim 0.5 \%$. Following incubation, the medium was collected and centrifuged at $1000 \times g$ for 10 minutes at $4{ }^{\circ} \mathrm{C}$, after which the supernatant was transferred to a new tube. Similarly, normoxic conditioned medium (NCM) was derived from hMSCcultured media under normoxic conditions for 12 hours with $5 \mathrm{ml} \mathrm{EBM-2.} \mathrm{Each} \mathrm{medium} \mathrm{was} \mathrm{stored} \mathrm{at}-80{ }^{\circ} \mathrm{C}$ until used.

\section{Lymphatic endothelial cells Proliferation assay}

Cell proliferation was measured using a Cell Counting Kit-8 (Dojindo, Kumamoto, Japan). hLECs were seeded 
at $5 \times 10^{3}$ cells per well in 96 -well culture plates and then cultured for 1 day. hLECs were then incubated with fresh EBM-2 for 12 hours. After the medium was removed, cells were washed twice with phosphate-buffered saline (PBS). Each group was subsequently treated with EBM-2, NCM, or HCM, respectively, after which the cells were incubated for 24 hours in a $37^{\circ} \mathrm{C}$ humidified atmosphere incubator containing $5 \% \mathrm{CO}_{2}$. Following incubation, Cell Counting Kit- 8 was added to each well and samples were then incubated for 2 hours. Finally, the absorbance of water soluble formazan dye was measured at $450 \mathrm{~nm}$ using a microplate reader (Molecular Devices, Sunnyvale, CA, USA). All experiments were performed in triplicate.

\section{Migration assay}

hLECs $\left(2 \times 10^{4}\right.$ cells) were seeded into the upper chamber of a Transwell filter with $8 \mu \mathrm{m}$ pores (Costar Corning, New York, NY, USA) coated with $10 \mu \mathrm{g} / \mathrm{ml}$ fibronectin. They were deprived of serum for 12 hours with EBM-2, after which EBM-2, NCM, or HCM were added to the lower chamber. Cells on the upper chambers were incubated at $37{ }^{\circ} \mathrm{C}$ for 9 hours under different stimuli. Following incubation, cells on the underside of the filter were stained with $0.25 \%$ crystal violet. Nonmigrating cells on the upper side of the filter were removed with cotton swabs. The filter was then photographed using a digital microscope camera system (Olympus, Shinjuku, Japan), or stained cells were dissolved in $10 \%$ acetic acid and transferred to a 96-well plate for colorimetric reading at $560 \mathrm{~nm}$ using a microplate reader (Molecular Devices).

\section{Immunoblot analysis}

hLECs were washed twice in ice-cold PBS, then lysed with lysis buffer (Cell Signaling, Danvers, MA, USA) containing the protease inhibitor cocktail PhosSTOP (Roche, Basel, Switzerland), and incubated at $4{ }^{\circ} \mathrm{C}$ for 30 minutes. Protein concentrations were determined using a BCA protein assay kit (Thermo Fisher Scientific, Inc., Waltham, MA, USA), after which they were separated in $10 \%$ SDS-polyacrylamide gel and transferred to PVDF membrane (Millipore, Billerica, MA, USA). The membrane was then blocked using TBS-T (0.1\% Tween 20) containing $5 \%$ $(\mathrm{w} / \mathrm{v})$ bovine serum albumin (BSA) for 1 hour at room temperature. The membranes were then washed with TBS-T and incubated with primary antibody overnight at $4{ }^{\circ} \mathrm{C}$. The next morning, the membrane was washed three times with TBS-T for 5 minutes each and incubated with horseradish peroxidase-conjugated secondary antibody for 1 hour at room temperature. After extensive washing, bands were detected using enhanced chemiluminescence reagent (AbClon, Seoul, Republic of Korea) and band intensities were measured using a Photo-Image System (Molecular Dynamics, Sunnyvale, CA, USA). MFN1
(Novus, Littleton, CO, USA), MFN2 (Sigma, St. Louis, MO, USA), extracellular signal regulated kinase (ERK; Santa Cruz, CA, USA), p-ERK (Santa Cruz), lymphatic vessel endothelial hyaluronan receptor 1(LYVE-1; Novus), and beta-actin (Sigma) antibodies were used in the experiment.

\section{Real-time $P C R$}

Total RNA was isolated using TRIzol ${ }^{\circ}$ Reagent (Life Technologies, Waltham, MA, USA). The total RNA quality and concentration were measured using NanoDrop Lite (Thermo Fisher Scientific, Inc.). Single-stranded cDNA was synthesized from total RNA using a reverse transcription system (Promega, Fitchburg, WI, USA) according to the product guidelines. Amplification and detection of specific products were performed in a StepOnePlus Real-time PCR System (Life Technologies) using a FastStart Essential DNA Green Master (Roche). PCR conditions consisted of $95^{\circ} \mathrm{C}$ for 10 minutes followed by 40 cycles of $95^{\circ} \mathrm{C}$ for 10 seconds and $60^{\circ} \mathrm{C}$ for $10 \mathrm{sec}-$ onds. The threshold cycle $(\mathrm{Ct})$ of each target gene was automatically defined and normalized to the control glyceraldehyde 3-phosphate dehydrogenase (GAPDH) $(\Delta \mathrm{Ct}$ value). The relative differences in the expression levels of each mRNA in VSMCs $\left({ }^{\Delta \Delta} \mathrm{Ct}\right)$ were calculated and presented as fold induction $\left(2^{-\Delta \Delta} \mathrm{Ct}\right)$. Real-time PCR primers consisted of the following groups: EGF forward primer, 5'-GGT CTT GCT GTG GAC TGG AT-3'; EGF reverse primer, 5'-CTG CTA CAG CAA ATG GGT GA-3'; IGF1 forward primer, 5'-TCA CCT TCA CCA GCT CTG C3'; IGF-1 reverse primer, 5' -TGG TAG ATG GGG GCT GAT AC-3'; HGF forward primer, 5'-GCC TGA AAG ATA TCC CGA CA-3'; HGF reverse primer, 5'-GCC ATT CCC ACG ATA ACA AT-3'; FGF-2 forward primer, 5'-AGC GGC TGT ACT GCA AAA AC-3'; FGF reverse primer, 5'-CTT TCT GCC CAG GTC CTG TT-3'; VEGF-A forward primer, 5'-AGT CCA ACA TCA CCA TGC AG-3'; VEGF-A reverse primer, 5' -TTC CCT TTC CTC GAA CTG ATT T-3'; VEGF-C forward primer, 5' CCT CAA CTC AAG GAC AGA AGA G-3'; VEGF-C reverse primer, 5 '-CTG GCA GGG AAC GTC TAA TAA T-3'; GAPDH forward primer, 5'-ACA TCG CTC AGA CAC CAT G-3'; and GAPDH reverse primer, 5'-TGT AGT TGA GGT CAA TGA AGG G-3'.

\section{Tube formation assay}

A Matrigel-based tube formation assay was performed. Each well of a 96-well culture plate was coated with $50 \mu \mathrm{l}$ of ECMatrix ${ }^{\text {tix }}$ (Millipore) and then allowed to incubate for 1 hour at $37^{\circ} \mathrm{C}$. Next, hLECs were seeded onto the coated wells at a density of $1 \times 10^{4}$ cells/well, cultured with $500 \mu \mathrm{l}$ of EBM-2, NCM, or HCM, and incubated at $37{ }^{\circ} \mathrm{C}$ under $5 \% \mathrm{CO}_{2}$ for 12 hours. Following incubation, tube 
formation images were captured using a digital microscope camera system (Olympus).

\section{Hind limb mouse model of lymphedema}

The hind limb mouse model of lymphedema was obtained as described previously [11]. Eight-week-old BALB/c mice (Orient Bio Co., Seongnam, Korea) were assigned into groups of three. Normal mice were then anesthetized via subcutaneous injection of zoletil $(4 \mathrm{mg} /$ $\mathrm{kg})$ with rompun $(20 \mathrm{mg} / \mathrm{kg})$. After a mouse was fully anesthetized, $0.5 \%$ Evans blue solution was intradermally injected into the footpad of the hind limb to visualize the lymphatic vessels. A circumferential incision of the limb (thigh) was made to access lymph vessels that were subsequently electrocauterized (Bovie Medical Corporation, Clearwater, FL, USA). EBM-2, $\mathrm{NCM}$, and HCM were then subcutaneously injected at the site of the damaged area on the second day after preparing the edema models and then every 3 days after that for a period of 24 days. The injection volume was $100 \mu \mathrm{l}$ of 1 -in-50 concentrated media. All animals were maintained under a 12-hour light-dark cycle condition and had free access to food and water. Experimental procedures were approved by the Committee for Care and Use of Laboratory Animals, Yonsei University College of Medicine, and performed in accordance with the Guidelines and Regulations for Animal Care.

\section{Histological analysis}

To measure lymphangiogenesis, mice were sacrificed 4 weeks after the surgery, after which a mouse's entire thigh region, including the site at which hMSCconditioned medium was injected, was dissected. The hind limbs were excised from the sacrificed mouse, washed with PBS to remove the blood, and then fixed in $10 \%$ formalin solution for 1 day at $4{ }^{\circ} \mathrm{C}$. Samples were subsequently treated with decalcification solution (CalciClear Rapid; National Diagnostics, Atlanta, GA, USA) for 1 week. Tissue sections were sequentially mounted onto gelatin-coated glass slides to ensure that different stains could be used on successive tissue sections sliced through the injured area. After the sections were deparaffinized and rehydrated, samples were blocked using $2.5 \%$ normal horse serum and incubated with an antiLYVE-1 antibody. A biotinylated pan-specific universal secondary antibody and streptavidin/peroxidase complex reagent were used to treat the sections. Sections were subsequently stained with an antibody using a DAB substrate kit (Vector, Burlingame, CA, USA). Counterstaining was performed using $1 \%$ methyl green, and dehydration was performed using $100 \%$ n-butanol, ethanol, and xylene before mounting in VectaMount Mounting Medium (Vector, Burlingame, CA, USA). A coverslip was placed on top of each section, and the sections were then observed using light microscopy. The images for hematoxylin and eosin ( $\mathrm{H} \& \mathrm{E})$ and LYVE-1 were obtained using virtual microscopy (BX51/dot Slide; Olympus). Images for LYVE-1 were detected using microscopy and transferred to a computer equipped with the MetaMorph software (version 4.6; Molecular Devices, Sunnyvale, CA, USA).

\section{In vivo visualized lymphatic vessels}

We observed the lymphatic vessels with fluorescence 3 weeks after surgery. The hind limb mouse model was anesthetized via a subcutaneous injection of zoletil $(4 \mathrm{mg} / \mathrm{kg})$ with rompun $(20 \mathrm{mg} / \mathrm{kg})$. After the mouse was fully anesthetized, $30 \mu \mathrm{l}$ of fluorescein isothiocyanate (FITC)-dextran solution $(1 \mathrm{mg} / \mathrm{ml})$ was intradermally injected into the footpad of the hind limb to visualize the lymphatic vessels, after which the lymphatic vessels were observed under a fluorescence microscope (SteREO Discovery V12; Zeiss, Oberkochen, Germany).

\section{Statistical analysis}

Data are expressed as mean \pm standard deviation (SD). Significance differences between groups were identified using Student's $t$ test. Comparisons between more than two groups were made with one-way analysis of variance using Bonferroni's correction. $p<0.05$ was considered statistically significant.

\section{Results}

HCM enhance lymphangiogenesis in hLECs

To investigate the ability of HCM to undergo lymphangiogenesis in vitro, we tested the proliferation, migration, and tube formation in hLECs. Under normal growth conditions, HCM did not cause any positive effects and was similar to NCM (data not shown). Therefore, hLECs were starved in serum-free EBM-2 culture for 12 hours to simulate in vivo conditions prior to $\mathrm{NCM}$ or $\mathrm{HCM}$ treatment, after which the effects of HCM on lymphangiogenesis were investigated. HCM markedly increased proliferation by up to twofold (Fig. 1a), as well as migration (Fig. 1b), compared with the control media and NCM. In addition, tube formation was promoted with HCM (Fig. 1c). We also examined LYVE-1 and ERK phosphorylation as proliferation markers, resulting in increased expression and phosphorylation under HCM treatment (Fig. 1d). The results showed that HCM have the ability to induce lymphangiogenesis in hLECs.

\section{hMSCs induce the expression of pro-lymphangiogenesis factors under hypoxia}

We next investigated several growth factors from hMSCs, which are known to induce lymphangiogenesis in hLECs. hMSCs exposed to 12 hours of hypoxia were lysed and used to estimate mRNA expression levels. The mRNA 
a

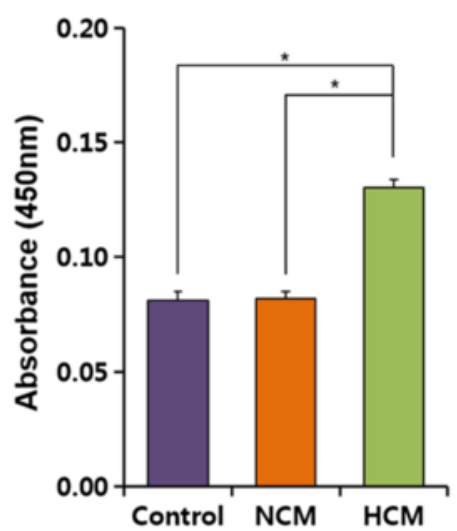

C
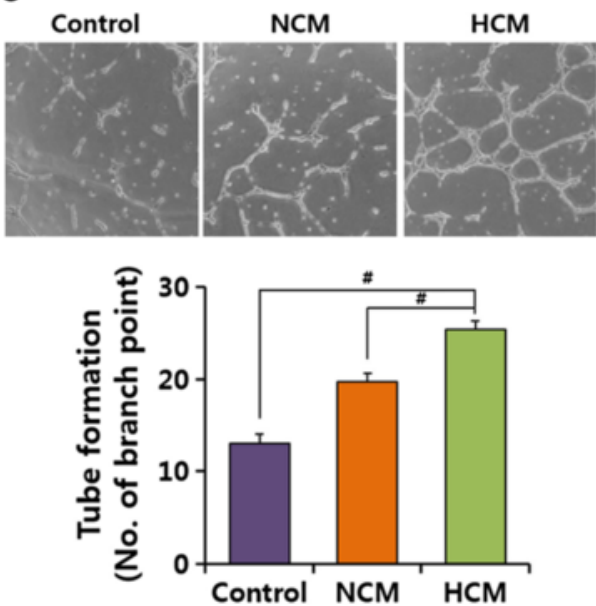
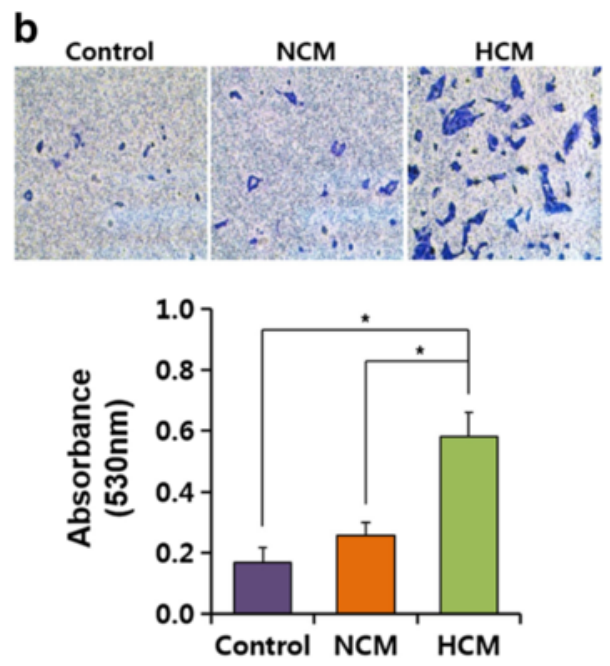

d

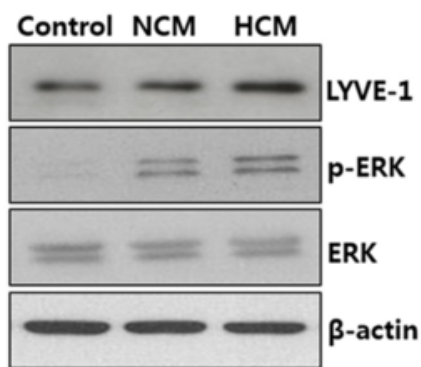

Fig. 1 hMSC-derived HCM stimulate hLECs in vitro. a hLEC proliferation was measured using the Cell Counting Kit-8 assay. b Representative images showed the results of migration assays of hLECs treated with NCM and HCM. c Representative photographs of tube formation in hLECs after treatment with NCM and HCM. $\mathbf{d}$ hLEC specific marker, LYVE-1, and phosphorylation of ERK were detected using western blot analysis. Values are represented as the mean of three measurements with the SD indicated by error bars. Control: EBM. ${ }^{*} p<0.001,{ }^{\#} p<0.05$. ERK extracellular signal regulated kinase, GAPDH glyceraldehyde 3-phosphate dehydrogenase, HCM hypoxic conditioned media, LYVE-1 lymphatic vessel endothelial hyaluronan receptor 1 , NCM normoxic conditioned media

expression of six different pro-lymphangiogenesis factors increased, with VEGF-A and VEGF-C showing significant increases in gene expression (Fig. 2a). Therefore, we further examined whether the expression of VEGF-A and VEGF-C (important lymphangiogenic-stimulating factors) increased in response to HCM in hLECs. Both factors markedly increased under HCM compared with NCM, which is the medium normally used for hMSC cultivation (Fig. 2b, c).

\section{HCM affect the expression of MFN1/2 in hLECs}

VEGF is known to induce MFNs in vascular endothelial cells but not in hLECs. Therefore, we examined expression levels of mRNA and protein of MFNs in hLECs under different conditions. HCM-treated cells showed the ability to induce both MFN1 and MFN2 expression compared with NCM-treated cells. NCM also increased MFN1 and MFN2 expression relative to the basal levels of these genes and proteins (Fig. $3 \mathrm{a}, \mathrm{b}$ ). We demonstrate the effect of lymphangiogenesis through experiments on an undisclosed MFN1/2 in hLECs. MFN1/2 small interfering RNAs (siRNAs) were used to demonstrate the relationship between MFN1/2 and lymphangiogenesis in the hLECs. Effects of MFN1/2 ablation were examined in hLECs using siRNA. Each siRNA was transfected into hLECs at a final concentration of $100 \mathrm{nM}$ for 2 days. Ablation of both MFN1 and MFN2 led to an approximately $93 \%$ and $80 \%$ reduction in MFN1 and in MFN2 


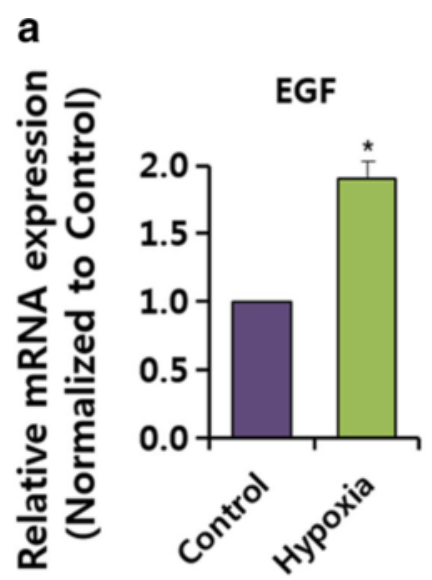

FGF-2

HGF
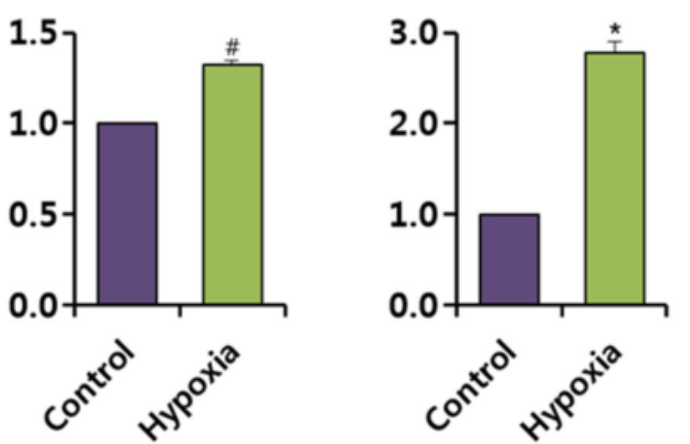

IGF-1

VEGF-A
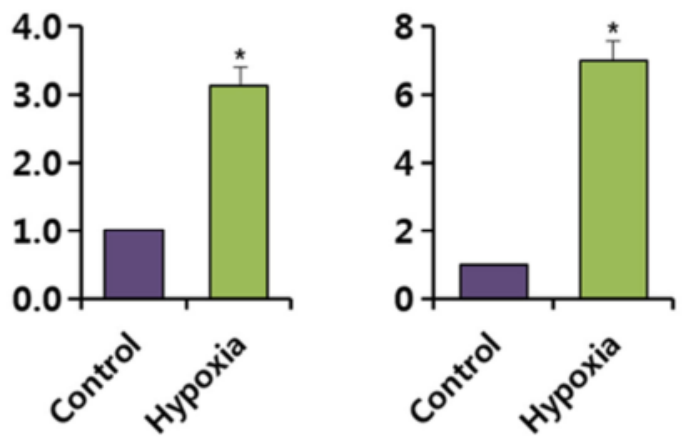

VEGF-C
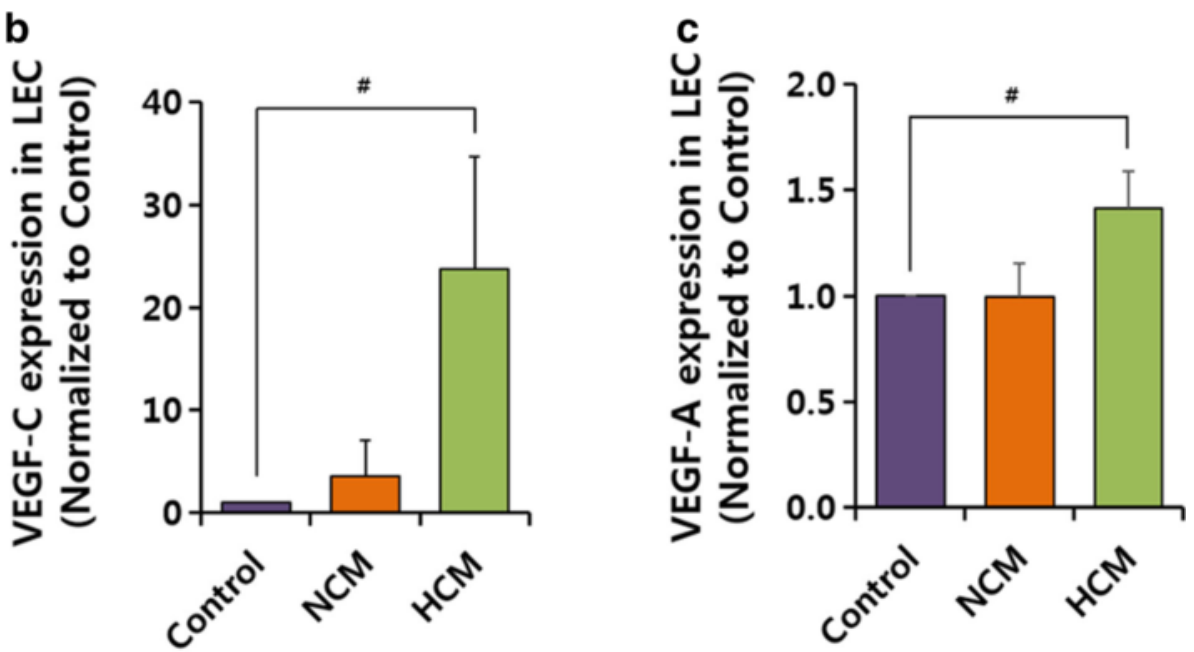

Fig. 2 Increased expression of lymphangiogenic factors of hMSCs in hypoxia. a Expression of lymphangiogenic factors (EGF, FGF, HGF, IGF-1, VEGF-A, and VEGF-C) of hMSCs in hypoxia. Expression of mRNAs was measured using quantitative RT-PCR. Gene expressions were normalized using GAPDH expression, respectively. b, c Expression of VEGF-C and VEGF-A was analyzed using quantitative RT-PCR in EBM, NCM, or HCMtreated hLECs. Control: EBM. Values are represented as the mean of three measurements with the SD indicated by error bars. ${ }^{*} p<0.001,{ }^{\#} p<0.05$. EGF epidermal growth factor, FGF fibroblast growth factor, HCM hypoxic conditioned media, HGF hepatocyte growth factor, IGF insulin-like growth factor, LEC lymphatic endothelial cells, NCM normoxic conditioned media, VEGF vascular endothelial growth factor 


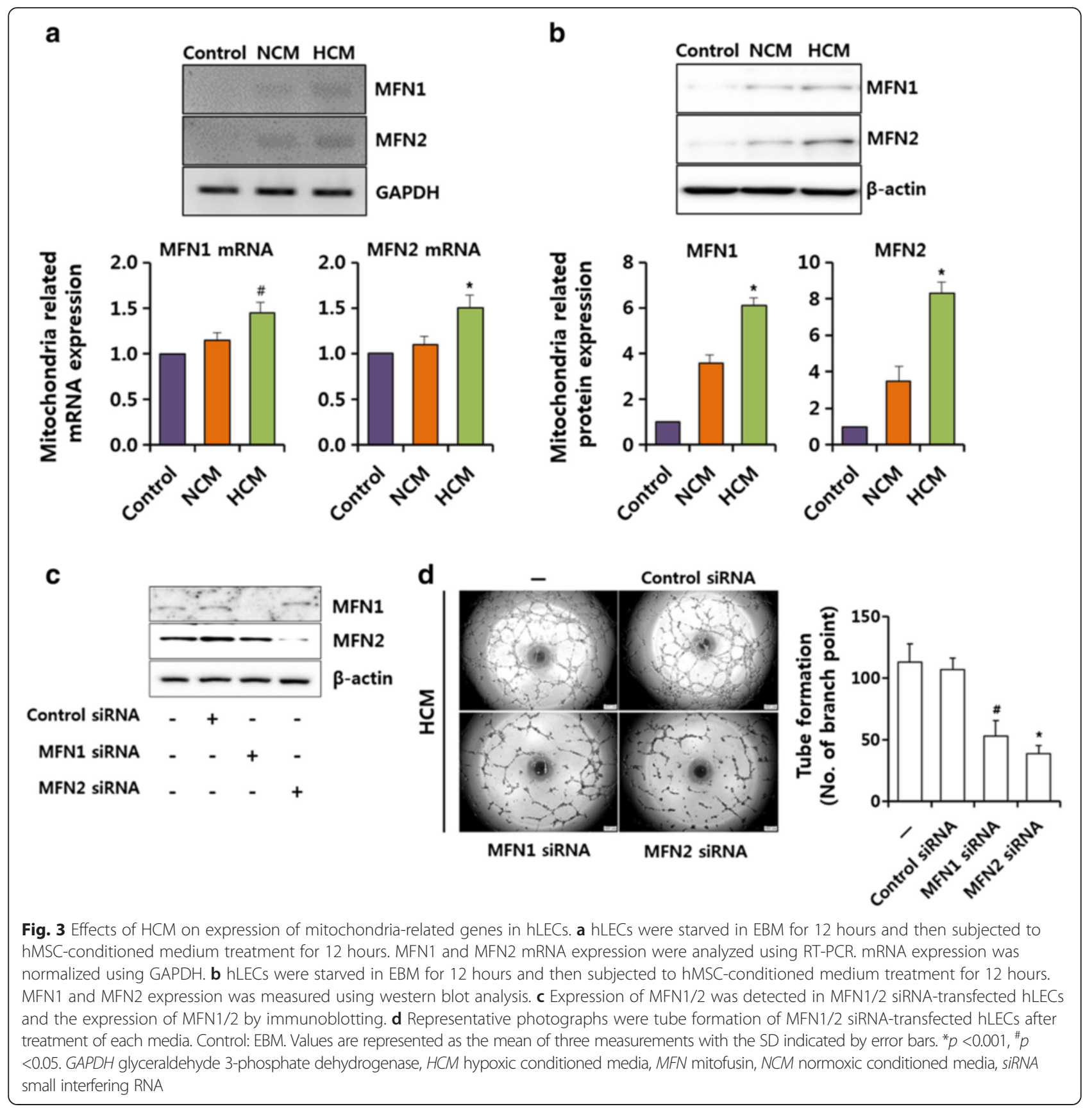

protein, respectively (Fig. 3c). Next, we detected the effects of MFN1/2 ablation on tube formation. As tube formation requires cellular migration on the Matrigel, we also investigated whether attenuation of MFN1/2 affects the ability for hLECs to migrate towards HCM. As a result, tube formation reduced in the MFN1 siRNA and MFN2 siRNA group compared with control group (Fig. 3d). We have identified the critical role of MFN1/2 in a positive effect on lymphangiogenesis by HCM from the results.
HCM promote in vivo lymphatic regeneration

To confirm the ability of HCM to induce lymphangiogenesis, a mouse model was generated using electrocauterization. The maximum swelling was examined 1-3 days after electrocauterization, and the thickness of the hind limb footpad was examined every 3 days for 27 days. As shown in Fig. 4a, footpad reduction was more effective in HCM-treated groups than in NCM-treated and control groups. $\mathrm{H}$ \& $\mathrm{E}$ staining showed histological edema changes, with the control group exhibiting large 

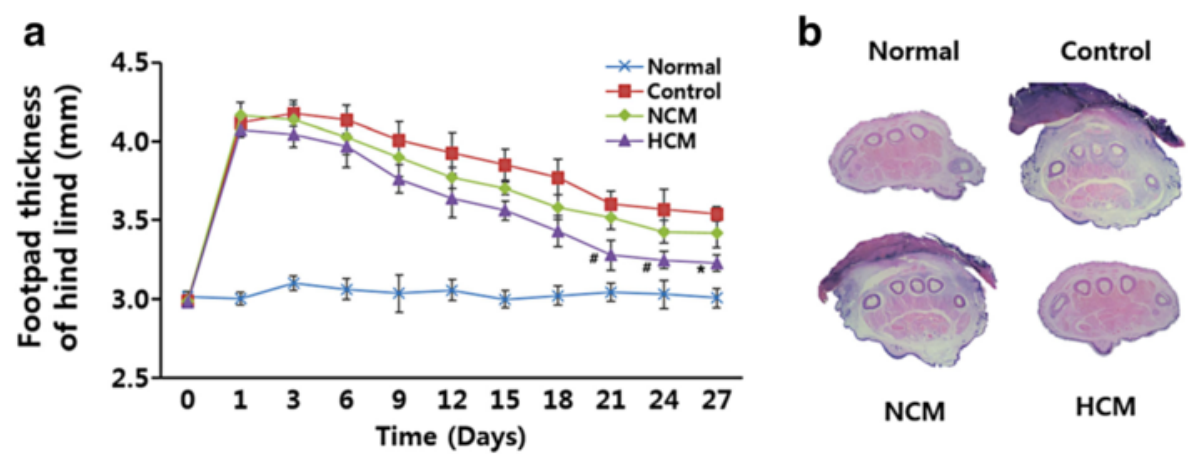

C

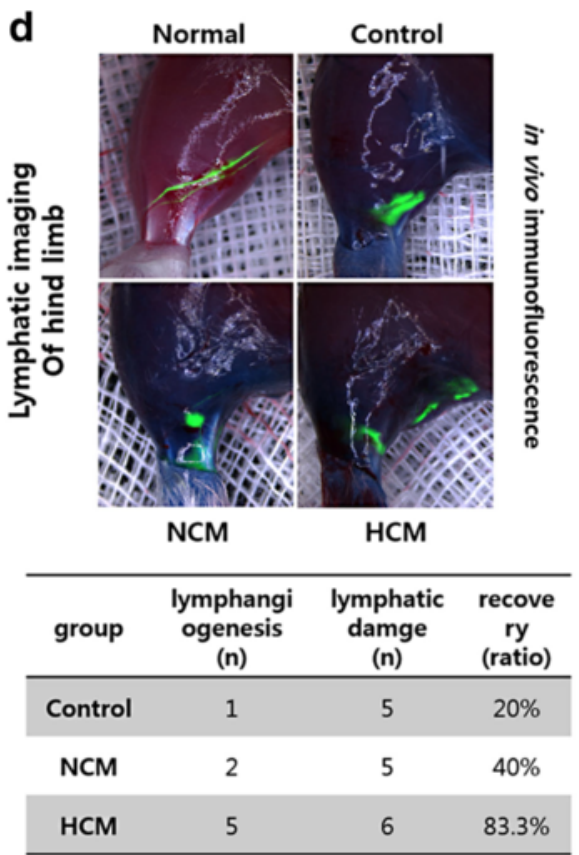

Fig. 4 Histological analyses of lymphangiogenesis. a Quantitative analysis showed significantly decreased footpad thickness in each group. b Fibrosis of the hind limb footpad was detected with $\mathrm{H}$ \& E stain. $\mathbf{c}$ Lymphatic endothelium marker, LYVE-1, was stained brown in HCM groups. Lymphatic vessels are stained brown (arrows). The scale bar represents $100 \mu \mathrm{m}$. d Fluorescence imaging of lymphatic vessels on mouse hind limbs after foot pad injection of FITC-dextran. Values are represented as the mean of three measurements with the SD indicated by error bars. ${ }^{*} p$ $<0.001, " \#<0.05$. HCM hypoxic conditioned media, LYVE-1 lymphatic vessel endothelial hyaluronan receptor 1, NCM normoxic conditioned media

cytosolic damage such as fibrosis. Although footpads from the NCM-treated groups showed less cytosolic damage, HCM-treated footpads showed marked recovery compared with normal footpads (Fig. 4b). Therefore, we further investigated whether these recovery effects were due to lymphatic regeneration. Each group of footpad samples was stained with LYVE-1, a marker of hLECs, and more LYVE-1-positive staining was observed in the HCM-treated group than in the control or NCMtreated groups (Fig. 4c). To confirm the regeneration effect of HCM in hLECs in vivo, we injected FITC-dextran to visualize the lymphatic vessels. NCM also showed an increased regeneration effect on lymphatic vessels of up to twofold when compared with that of the control, whereas
HCM showed a more significant lymphangiogenesispromoting ability than the control (Fig. 4d).

\section{Discussion}

This study demonstrated for the first time that HCM promote lymphangiogenesis by MFN1 and MFN2 regulation in hLECs. Furthermore, the study demonstrated the potential impact that lymphangiogenesis may have on damaged lymphatic vessel repair in vivo.

MSCs appear to be the most feasible, potentially safe, and effective stem cells for therapeutic lymphangiogenesis via direct differentiation toward lymphatic lineage and cytokine secretion $[4,21,32]$. Because 
cytokines have short-lasting efficacies, secretory modification is needed. Cytokine secretion of MSCs can be induced by hypoxia, inflammatory stimuli, and threedimensional culture configuration. Rehman et al. [33] demonstrated that paracrine factors released from stem cells under hypoxia demonstrated anti-apoptotic or pro-angiogenic effects. Several studies have reported that HCM contain cytokines and growth factors, such as EGF, FGF-2, HGF, IGF-1, VEGF-A, and VEGF-C [34, 35]. Several reports have also stated that these cytokines could augment lymphangiogenesis in animal models of lymphedema [5-10, 36]. Our results showed higher mRNA expressions of these cytokines in hMSC under hypoxia than under normoxic conditions (Fig 2; EGF, $p<0.001$; FGF-2, $p<0.05$; HGF, $p$ $<0.001$; IGF-1, $p<0.001$; VEGF-A, $p<0.001$; VEGF-C, $p<0.05)$.

We demonstrated that HCM has the ability to enhance hLEC proliferation and migration and to increase lymphatic vessel formation. Zhan et al. also showed that culture medium from hMSCs increased lymphatic vessel formation by inducing VEGF receptor-3 (VEGFR-3) in hLECs [37]. As shown in Fig. 2, increased mRNA expression of VEGF-A and VEGF-C by hypoxic hMSCs and HCM-treated hLECs was investigated. VEGFR-2 and VEGFR-3 are known to exist in hLECs and are activated by VEGF-A and VEGF-C, respectively [38, 39]. In addition, phosphorylation of ERK1/2 appears to be primarily caused by VEGF-A/VEGF receptor- 2 upon hMSC stimulation in hLECs. Shimizu et al. [32] found that the expression of $\mathrm{p}$-ERF was regulated by VEGF-C. We also showed that ERK phosphorylation and expression of LYVE-1 was increased by HCM in hLECs, which supports the increased VEGF-A expression in hLECs.

Mitochondrial function integrity is essential for organ homeostasis, particularly for rapidly dividing cells, such as hemopoietic precursor cells [40]. In this study, we ascertained the potential for HCM to promote lymphangiogenesis by regulating mitochondrial-related genes. Our results showed that mRNA expression and protein levels of MFN1 and MFN2 increased under HCM treatment in hLECs. Although the role of MFNs has been investigated in other cell types, such as endothelial cells, this is the first study, to the best of our knowledge, to investigate their roles in hLECs.

We focused on paracrine mediators of hMSCs cultured with HCM. Direct injection of HCM into a lymphedema mouse demonstrated lymphangiogenesis and edema improvement. As a precursor to future clinical trials, our study shows therapeutic potential for stimulation of lymphangiogenesis.

To use HCM, we should consider some additional details. According to a recent study [15] performed using hMSCs obtained from 20 people, there is a difference in endothelial differentiation ability of the hMSCs. Lin et al. [16] reported differences in expression of VEGF and interleukin-8 among three donors. We believe that the donor cell variation should be considered to be applicable for patient therapeutics. Furthermore, the half-life of cytokines and growth factors, which are included in the conditioned media, has been reported to be very short in vivo [41, 42]. For this reason, conditioned media must be more frequently administered. Nevertheless, $\mathrm{HCM}$ are a promising treatment for damaged tissue regeneration.

\section{Conclusions}

We showed the effects of HCM from hMSCs on in vitro and in vivo lymphangiogenesis. Our results demonstrated an increase in hMSC lymphangiogenic cytokines, which can lead to proliferation, migration, and tube formations in hLECs, thereby showing hMSCs derived HCM potential therapeutic effects in the lymphedema mouse model. In addition, expression of MFN1 and MFN2 is suggested for their possible roles in lymphangiogenesis. Treatment with HCM may thus be a therapeutic strategy for lymphedema.

\begin{abstract}
Abbreviations
Ct: Threshold cycle; EBM: Endothelial basal medium; EGF: Epidermal growth factor; ERK: Extracellular signal regulated kinase; FITC: Fluorescein isothiocyanate; FGF: Fibroblast growth factor; GAPDH: Glyceraldehyde 3phosphate dehydrogenase; HCM: Hypoxic conditioned media; H \& E: Hematoxylin and eosin; HGF: Hepatocyte growth factor; hLEC: Human lymphatic endothelial cell; hMSC: Human mesenchymal stem cell; IGF: Insulin-like growth factor; LYVE-1: lymphatic vessel endothelial hyaluronan receptor 1; MFN: Mitofusin; MSC: Mesenchymal stem cell; NCM: Normoxic conditioned media; PBS: Phosphate-buffered saline; ROS: Reactive oxygen species; SD: standard deviation; siRNA: Small interfering RNA; VEGF: Vascular endothelial growth factor; VEGFR: Vascular endothelial growth factor receptor.
\end{abstract}

\section{Competing interests}

The authors declare that they have no competing interests.

\section{Authors' contributions}

CYL and JYK carried out the experiments, designed the study, and drafted and revised the manuscript. SL and $\mathrm{OH}$ participated in the data analysis and drafted the manuscript. WC and D-HJ established the hypotheses, designed the study, and revised the manuscript. All authors agreed to be accountable for all aspects of the work in ensuring that questions related to the accuracy or integrity of any part of the work are appropriately investigated and resolved. All authors read and approved the manuscript.

\section{Acknowledgements}

This research was supported by the Catholic Medical Center Research Foundation made in the program year of 2013.

\section{Author details}

'Department of Integrated Omics for Biomedical Sciences, Graduate School, Yonsei University, Seoul 03722, Republic of Korea. ${ }^{2}$ Department of Rehabilitation Medicine, National Traffic Injury Rehabilitation Hospital, College of Medicine, The Catholic University of Korea, Yangpyeong-gun 12564, Republic of Korea. ${ }^{3}$ Institute for Bio-Medical Convergence, College of Medicine, Catholic Kwandong University, Gangneung-si 25601Gangwon-do, Republic of Korea. ${ }^{4}$ Catholic Kwandong University International St. Mary's Hospital, Incheon Metropolitan City 22711, Republic of Korea. ${ }^{5}$ Department of Biology Education, College of Education, Pusan National University, Busan 
46241, Republic of Korea. ${ }^{6}$ Department of Rehabilitation Medicine, Incheon St. Mary's Hospital, College of Medicine, The Catholic University of Korea, Dongsu-ro 56, Bupyeong-gu, Incheon 21431, Republic of Korea.

Received: 17 August 2015 Revised: 15 February 2016

Accepted: 22 February 2016

Published online: 11 March 2016

\section{References}

1. Oremus M, Walker K, Dayes I, Raina P. AHRQ technology assessments. Diagnosis and treatment of secondary lymphedema. Rockville: Agency for Healthcare Research and Quality (US); 2010.

2. Kerchner K, Fleischer A, Yosipovitch G. Lower extremity lymphedema update: pathophysiology, diagnosis, and treatment guidelines. J Am Acad Dermatol. 2008:59(2):324-31. doi:10.1016/j.jaad.2008.04.013.

3. Kim IG, Lee JY, Lee DS, Kwon JY, Hwang JH. Extracorporeal shock wave therapy combined with vascular endothelial growth factor-C hydrogel for lymphangiogenesis. J Vasc Res. 2013;50(2):124-33. doi:10.1159/000343699.

4. Qi S, Pan J. Cell-based therapy for therapeutic lymphangiogenesis. Stem Cells Dev. 2015;24(3):271-83. doi:10.1089/scd.2014.0390.

5. Marino D, Angehrn Y, Klein S, Riccardi S, Baenziger-Tobler N, Otto VI, et al. Activation of the epidermal growth factor receptor promotes lymphangiogenesis in the skin. J Dermatol Sci. 2013;71(3):184-94. doi:10. 1016/j.jdermsci.2013.04.024

6. Cao R, Bjorndahl MA, Gallego MI, Chen S, Religa P, Hansen AJ, et al. Hepatocyte growth factor is a lymphangiogenic factor with an indirect mechanism of action. Blood. 2006;107(9):3531-6. doi:10.1182/blood2005-06-2538.

7. Chang LK, Garcia-Cardena G, Farnebo F, Fannon M, Chen EJ, Butterfield C, et al. Dose-dependent response of FGF-2 for lymphangiogenesis. Proc Natl Acad Sci U S A. 2004;101(32):1 1658-63. doi:10.1073/pnas.0404272101.

8. Yoon YS, Murayama T, Gravereaux E, Tkebuchava T, Silver M, Curry C, et al. VEGF-C gene therapy augments postnatal lymphangiogenesis and ameliorates secondary lymphedema. J Clin Invest. 2003;111(5):717-25. doi:10.1172/jci15830.

9. Karkkainen MJ, Haiko P, Sainio K, Partanen J, Taipale J, Petrova TV, et al. Vascular endothelial growth factor $C$ is required for sprouting of the first lymphatic vessels from embryonic veins. Nat Immunol. 2004;5(1):74-80. doi:10.1038/ni1013.

10. Cursiefen C, Chen L, Borges LP, Jackson D, Cao J, Radziejewski C, et al. VEGF-A stimulates lymphangiogenesis and hemangiogenesis in inflammatory neovascularization via macrophage recruitment. J Clin Invest. 2004;113(7):1040-50. doi:10.1172/jci20465.

11. Hwang JH, Kim IG, Lee JY, Piao S, Lee DS, Lee TS, et al. Therapeutic lymphangiogenesis using stem cell and VEGF-C hydrogel. Biomaterials. 2011;32(19):4415-23. doi:10.1016/j.biomaterials.2011.02.051.

12. Ryan JM, Barry FP, Murphy JM, Mahon BP. Mesenchymal stem cells avoid allogeneic rejection. J Inflamm (Lond). 2005;2:8. doi:10.1186/1476-9255-2-8.

13. Zhang M, Methot D, Poppa V, Fujio Y, Walsh K, Murry CE. Cardiomyocyte grafting for cardiac repair: graft cell death and anti-death strategies. J Mol Cell Cardiol. 2001;33(5):907-21. doi:10.1006/jmcc.2001.1367.

14. Nygren JM, Jovinge S, Breitbach M, Sawen P, Roll W, Hescheler J, et al. Bone marrow-derived hematopoietic cells generate cardiomyocytes at a low frequency through cell fusion, but not transdifferentiation. Nat Med. 2004; 10(5):494-501. doi:10.1038/nm1040.

15. Portalska KJ, Groen N, Krenning G, Georgi N, Mentink A, Harmsen MC, et al. The effect of donor variation and senescence on endothelial differentiation of human mesenchymal stromal cells. Tissue Eng Part A. 2013;19(21-22): 2318-29. doi:10.1089/ten.TEA.2012.0646.

16. Lin X, Robinson M, Petrie T, Spandler V, Boyd WD, Sondergaard CS. Small intestinal submucosa-derived extracellular matrix bioscaffold significantly enhances angiogenic factor secretion from human mesenchymal stromal cells. Stem Cell Res Ther. 2015;6:164. doi:10.1186/s13287-015-0165-3.

17. Joyce N, Annett G, Wirthlin L, Olson S, Bauer G, Nolta JA. Mesenchymal stem cells for the treatment of neurodegenerative disease. Regen Med. 2010;5(6):933-46. doi:10.2217/Rme.10.72.

18. Madrigal M, Rao KS, Riordan NH. A review of therapeutic effects of mesenchymal stem cell secretions and induction of secretory modification by different culture methods. J Transl Med. 2014;12:260. doi:10.1186/s12967-014-0260-8.
19. Baraniak PR, McDevitt TC. Stem cell paracrine actions and tissue regeneration. Regen Med. 2010;5(1):121-43. doi:10.2217/Rme.09.74.

20. Maertens L, Erpicum C, Detry B, Blacher S, Lenoir B, Carnet O, et al. Bone marrow-derived mesenchymal stem cells drive lymphangiogenesis. Plos One. 2014;9(9):e106976. doi:10.1371/journal.pone.0106976.

21. Conrad C, Niess H, Huss R, Huber S, von Luettichau I, Nelson PJ, et al. Multipotent mesenchymal stem cells acquire a lymphendothelial phenotype and enhance lymphatic regeneration in vivo. Circulation. 2009; 119(2):281-9. doi:10.1161/CIRCULATIONAHA.108.793208.

22. Gil-Ortega M, Garidou L, Barreau C, Maumus M, Breasson L, Tavernier G, et al. Native adipose stromal cells egress from adipose tissue in vivo: evidence during lymph node activation. Stem Cells. 2013;31(7):1309-20. doi:10.1002/stem.1375.

23. Chen L, Xu Y, Zhao J, Zhang Z, Yang R, Xie J, et al. Conditioned medium from hypoxic bone marrow-derived mesenchymal stem cells enhances wound healing in mice. PLoS One. 2014;9(4):e96161. doi:10.1371/journal. pone. 0096161 .

24. Burlacu A, Grigorescu G, Rosca AM, Preda MB, Simionescu M. Factors secreted by mesenchymal stem cells and endothelial progenitor cells have complementary effects on angiogenesis in vitro. Stem Cells Dev. 2013;22(4): 643-53. doi:10.1089/scd.2012.0273.

25. Berridge MJ, Lipp P, Bootman MD. The versatility and universality of calcium signalling. Nat Rev Mol Cell Biol. 2000;1 (1):11-21. doi:10.1038/35036035.

26. Green DR, Reed JC. Mitochondria and apoptosis. Science. 1998;281(5381): 1309-12. doi:10.1126/science.281.5381.1309.

27. Turrens JF. Mitochondrial formation of reactive oxygen species. J Physiol Lond. 2003;552(2):335-44. doi:10.1113/jphysiol.2003.049478.

28. Song ZY, Ghochani M, McCaffery JM, Frey TG, Chan DC. Mitofusins and OPA1 mediate sequential steps in mitochondrial membrane fusion. Mol Biol Cell. 2009;20(15):3525-32. doi:10.1091/mbc.E09-03-0252.

29. Han XJ, Lu YF, Li SA, Kaitsuka T, Sato Y, Tomizawa K, et al. CaM kinase I alpha-induced phosphorylation of Drp1 regulates mitochondrial morphology. J Cell Biol. 2008;182(3):573-85. doi:10.1083/jcb.200802164.

30. Zhou Q, Gensch C, Keller C, Schmitt H, Esser J, Moser M, et al. MnTBAP stimulates angiogenic functions in endothelial cells through mitofusin-1. Vasc Pharmacol. 2015;72:163-71. doi:10.1016/j.vph.2015.05.007.

31. Lugus JJ, Ngoh GA, Bachschmid MM, Walsh K. Mitofusins are required for angiogenic function and modulate different signaling pathways in cultured endothelial cells. J Mol Cell Cardiol. 2011;51(6):885-93. doi:10.1016/j.yjmcc. 2011.07.023

32. Shimizu Y, Shibata R, Shintani S, Ishii M, Murohara T. Therapeutic lymphangiogenesis with implantation of adipose-derived regenerative cells. J Am Heart Assoc. 2012;1(4):e000877. doi:10.1161/jaha.112.000877.

33. Rehman J, Traktuev D, Li J, Merfeld-Clauss S, Temm-Grove CJ, Bovenkerk JE, et al. Secretion of angiogenic and antiapoptotic factors by human adipose stromal cells. Circulation. 2004;109(10):1292-8. doi:10.1161/01.CIR. 0000121425.42966.F1.

34. Crisostomo PR, Wang Y, Markel TA, Wang M, Lahm T, Meldrum DR. Human mesenchymal stem cells stimulated by TNF-alpha, LPS, or hypoxia produce growth factors by an NF kappa B- but not JNK-dependent mechanism. Am J Physiol Cell Physiol. 2008;294(3):C675-82. doi:10.1152/ajpcell.00437.2007.

35. Efimenko A, Starostina E, Kalinina N, Stolzing A. Angiogenic properties of aged adipose derived mesenchymal stem cells after hypoxic conditioning. J Transl Med. 2011;9:10. doi:10.1186/1479-5876-9-10.

36. Bjorndahl M, Cao R, Nissen LJ, Clasper S, Johnson LA, Xue Y, et al. Insulinlike growth factors 1 and 2 induce lymphangiogenesis in vivo. Proc Natl Acad Sci U S A. 2005;102(43):15593-8. doi:10.1073/pnas.0507865102.

37. Zhan J, Li YH, Yu J, Zhao YY, Cao WM, Ma J, Sun XX, Sun L, Qian H, Zhu W, Xu WR. Culture medium of bone marrow-derived human mesenchymal stem cells effects lymphatic endothelial cells and tumor lymph vessel formation. Oncol Lett. 2015;9:1221-1226. doi: 10.3892/OI.2015.2868

38. Religa P, Cao RH, Bjorndahl M, Zhou ZJ, Zhu ZP, Cao YH. Presence of bone marrow-derived circulating progenitor endothelial cells in the newly formed lymphatic vessels. Blood. 2005;106(13):4184-90. doi: 10.1182/blood-2005-010226.

39. Dellinger MT, Brekken RA. Phosphorylation of Akt and ERK $1 / 2$ is Required for VEGF-ANEGFR2-Induced Proliferation and Migration of Lymphatic Endothelium. Plos One. 2011;6(12):e28947. doi: 10.1371/journal.pone. 0028947.

40. Sack MN. Mitofusin function is dependent on the distinct tissue and organ specific roles of mitochondria. Mol Cell Cardiol. 2011;51(6):881-2. doi: 10. 1016/j.yjmcc.2011.09.004. 
41. Yde P, Mengel B, Jensen MH, Krishna S, Trusina A. Modeling the NF-kappaB mediated inflammatory response predicts cytokine waves in tissue. BMC Syst Biol. 2011;5:115. doi: 10.1186/1752-0509-5-115.

42. Khosravi A, Cutler CM, Kelly MH, Chang R, Royal RE, Sherry RM, et al.

Determination of the elimination half-life of fibroblast growth factor-23. J Clin Endocrinol Metab. 2007;92(6):2374-7. doi:10.1210/jc.2006-2865.

Submit your next manuscript to BioMed Central and we will help you at every step:

- We accept pre-submission inquiries

- Our selector tool helps you to find the most relevant journal

- We provide round the clock customer support

- Convenient online submission

- Thorough peer review

- Inclusion in PubMed and all major indexing services

- Maximum visibility for your research

Submit your manuscript at www.biomedcentral.com/submit 